\title{
Magnetization in quasiperiodic magnetic multilayers with biquadratic exchange and uniaxial anisotropy
}

\author{
C.G. Bezerra and M.G. Cottam \\ Department of Physics and Astronomy, University of Western Ontario, N6A 3K7, \\ London, Ontario, Canada.
}

\begin{abstract}
A theoretical study is made of the magnetization versus applied field curves of ferromagnetic/nonmagnetic multilayers constructed according to a Fibonacci quasiperiodic sequence. The ferromagnetic films are assumed to have uniaxial anisotropy and are coupled by both bilinear and biquadratic effective exchange. The effects of quasiperiodicity in the magnetic phases are illustrated numerically for $\mathrm{Fe} / \mathrm{Cr}$ systems.
\end{abstract}

PACS: 71.70.Gm; 75.70.Ak; 75.70.-i; 71.23.Ft

Keywords: Quasicrystals; Exchange coupling - biquadratic; Thin films - multilayer; Anisotropy - uniaxial

Magnetic multilayers have attracted attention due to their physical properties and potential for technological applications [1]. The coupling between the magnetic layers often corresponds to a bilinear (BL), or Heisenberg, exchange type. However, some multilayers also exhibit a biquadratic (BQ) exchange coupling, which favours a $90^{\circ}$ orientation between the adjacent ferromagnetic layers $[2,3]$. The BQ exchange, although often weaker than the BL exchange, can be large in some systems. This leads to new possibilities for the phase diagrams and magnetization curves.

Furthermore, it is possible to build magnetic multilayers where the film thickness and stacking are well controlled, as in periodic superlattices. However, there has also been interest in magnetic systems formed with the layers following a quasiperiodic sequence. Recent studies, applied to Fe/Cr Fibonacci multilayers, showed that a self-similar behaviour of the magnetic properties occurs only when the BQ and BL exchange are comparable $[4,5]$. The authors 
included effects of a cubic anisotropy (see, e.g., [6]). However, some magnetic multilayers may instead exhibit uniaxial anisotropy [7], and this gives a new symmetry to the system that, together with the BQ exchange and quasiperiodicity, may lead to new configurations. This has motivated the present work.

A Fibonacci structure is formed by juxtaposing two basic building blocks, $A$ and $B$, following a Fibonacci sequence. For the present work we choose ferromagnetic Fe and nonmagnetic $\mathrm{Cr}$ to correspond to $A$ and $B$, respectively. By definition, the $N t h$ Fibonacci generation, $S_{N}$, is obtained by appending generation $N-2$ to generation $N-1$, i.e., $S_{N}=S_{N-1} S_{N-2}$ taking $S_{0}=B$ and $S_{1}=A$. Thus, for example, $S_{3}$ and $S_{5}$ correspond to the magnetic multilayers $\mathrm{Fe} / \mathrm{Cr} / \mathrm{Fe}$ and $\mathrm{Fe} / \mathrm{Cr} / \mathrm{Fe} / \mathrm{Fe} / \mathrm{Cr} / \mathrm{Fe} / \mathrm{Cr} / \mathrm{Fe}$ respectively.

We assume the magnetization of the ferromagnetic layers to be in the $x y$ plane and take the $z$-axis as the growth direction. Following $[4,5]$ but with the anisotropy replaced by a uniaxial term as in [7], the magnetic energy per unit area is

$$
\begin{aligned}
& \frac{E_{T}}{t M_{S}}=\sum_{i=1}^{n}\left(t_{i} / t\right)\left\{-H_{0} \cos \left(\theta_{i}-\theta_{H}\right)-\frac{1}{2} H_{u a} \cos ^{2}\left(\theta_{i}-\theta_{u a}\right)\right\} \\
& +\sum_{i=1}^{n-1}\left\{-H_{b l} \cos \left(\theta_{i}-\theta_{i+1}\right)+H_{b q} \cos ^{2}\left(\theta_{i}-\theta_{i+1}\right)\right\} .
\end{aligned}
$$

Index $i(=1,2, \ldots, n)$ labels the Fe layers in the $N$ th generation structure. For example, $n=4$ in the case of $N=5$, with three Fe layers having thickness $t$ and one having thickness $2 t$, where $t$ denotes the thickness of a single $A$ layer (the basic tile). In layer $i$ (with thickness $t_{i}$ ) the equilibrium magnetization is in the $x y$-plane at angle $\theta_{i}$ to the $x$-axis. The first term in eq. (1) is the Zeeman energy due to an applied field $H_{0}$ at an angle $\theta_{H}$. The second term is due to a uniaxial anisotropy $H_{u a}$ with easy axis specified by angle $\theta_{u a}$. The third and fourth terms (involving parameters $H_{b l}, H_{b q}$ ) describe the BL and BQ exchange between adjacent layers. The set $\{\theta\}_{i}$ of equilibrium angles is calculated numerically by minimizing the magnetic energy given by eq. (1). For that purpose we use two methods, namely, the simulated annealing and the gradient methods (see [5] for descriptions). The simulated annealing method is based on the fact that heating and then cooling a material slowly brings it into a more uniform state, which is the minimum energy state. In this process, the role played by a pseudo temperature $T$ is to allow the configurations to reach higher energy states with probability $p$ given by the Boltzmann law $p=\exp (-\Delta E / k T)$, where $\Delta E$ is the energy difference. Energy barriers that would otherwise force the configurations into local minima can then be overcome. On the other hand, the gradient method is based on finding the directional derivative of the magnetic energy in the search for its global min- 
imum in the $\mathrm{n}$-dimensional space composed of the variables $\{\theta\}_{i}$. It is the gradient of the magnetic energy with relation to the angles that furnishing the direction, and eventually the location, of the required global minimum. Both cited methods are used for each value of the applied magnetic field and for each set of magnetic parameters. We choose the configuration with the lowest energy furnished by both methods as giving the equilibrium configuration $\{\theta\}_{i}$. From the calculated $\{\theta\}_{i}$, we obtain normalized values of the net magnetization in the $H_{0}$ direction, appropriate to the $\mathrm{Fe} / \mathrm{Cr}$ system.

Magnetization curves found for $N=3,5$ and 7 are shown in Fig. 1. Here the in-plane magnetic field is applied along the easy axis. The magnetic parameters are such that $H_{b q}<<\left|H_{b l}\right|$ and $H_{b q}<<H_{u a}$. For $N=3$ (see Fig. 1a) the magnetizations in adjacent layers are antiparallel at low fields. As $H_{0}$ increases, a first-order phase transition occurs (at $\sim 0.8 \mathrm{kOe}$ ) to a spin-flop phase. Saturation is reached at $H_{0} \sim 1.9$ kOe. For $N=5$ (see Fig. 1b), due to the different thickness of the Fe layers, the net magnetization at low fields has about $20 \%$ of its saturation value. There are first-order phase transitions at $H_{0} \sim 1.0 \mathrm{kOe}$ and $H_{0} \sim 1.6 \mathrm{kOe}$. Saturation is reached at $\sim 2.9 \mathrm{kOe}$. When $N=7$ (Fig. 1c) there is again a non-zero magnetization at low fields. There are three first-order phase transitions at $H_{0} \sim 0.5 \mathrm{kOe}, 1.0 \mathrm{kOe}$ and $1.6 \mathrm{kOe}$.

Another set of magnetization curves is shown in Fig. 2, taking a larger BQ exchange and other parameters as before. For $N=3$, due to the strong $H_{b q}$ the adjacent layer magnetizations are only approximately antiparallel at low field (corresponding to an asymmetric phase [7]). A first-order phase transition occurs at $H_{0} \sim 0.4 \mathrm{kOe}$ to a spin-flop phase and saturation is reached at $H_{0} \sim 4.3 \mathrm{kOe}$. For $N=5$ all transitions appear continuous (second-order phase transitions) and saturation is reached at $H_{0} \sim 6.4 \mathrm{kOe}$. For $N=7$ there appears to be a first-order phase transition at $H_{0} \sim 0.15 \mathrm{kOe}$ and saturation is reached at $H_{0} \sim 6.6 \mathrm{kOe}$. Again the fifth and seventh generations exhibit a nonzero magnetization in the low-field region.

We have also studied cases where the applied field does not lie along the easy axis $\left(\theta_{H} \neq \theta_{u a}\right)$, and an example for $N=3$ is shown in Fig. 3 . When the field is applied at $45^{\circ}$ and $90^{\circ}$ from the easy axis, the magnetizations continuously rotate towards the field direction. There is no first-order phase transition in these configurations. The saturation fields for $45^{\circ}$ and $90^{\circ}$ are $H_{0} \sim 2.3 \mathrm{kOe}$ and $2.9 \mathrm{kOe}$, respectively.

In conclusion, we have modelled the magnetization versus applied field curves of Fe/Cr Fibonacci multilayers including both BL and BQ exchange. The role of uniaxial anisotropy is studied, including situations when the applied field direction is different from the easy axis. One can see a discrete self-similar pattern in the system when $H_{b q}<<\left|H_{b l}\right|$ (Fig. 1) and most transitions are of the first-order type. This property is highlighted by the boxes drawn as 
insets in Fig. 1. Specifically, the magnetization curves of higher generation $N$ reproduce some aspects of the magnetization curves of lower generation $N-2$. By contrast, when $H_{b q} \sim\left|H_{b l}\right|$ and most of the transitions are secondorder type, there is no apparent self-similarity. This behaviour, regarding the effect of BQ exchange, differs from that in quasiperiodic multilayers having cubic anisotropy $[4,5]$. We may surmise that the lower symmetry of uniaxial anisotropy changes the nature of the phase transitions and consequently the conditions for a self-similar pattern to occur in the magnetization curves. An analogous behaviour may also apply for the transport properties of the system and this provides a topic for future studies.

We gratefully acknowledges a fellowship (to CGB) from the Brazilian Research Council CNPq and partial financial support (to MGC) from NSERC of Canada.

\section{References}

[1] See, e.g., B. Heinrich and J.A.C. Bland (Eds.), Ultrathin Magnetic Structures, vol I and II, Springer, Berlin, 1994.

[2] M. Ruhrig, R. Schafer, A. Hubert, R. Mosler, J.A. Wolf, S. Demokritov and P. Grünberg, Phys. Status Solidi A 125 (1991) 635.

[3] For a recent review see S.O. Demokritov, J. Phys. D: Appl. Phys. 31 (1998) 925.

[4] C.G. Bezerra, J.M. de Araújo, C. Chesman and E.L. Albuquerque, Phys. Rev. B 60 (1999) 9264.

[5] C.G. Bezerra, J.M. de Araújo, C. Chesman and E.L. Albuquerque, J. Appl. Phys. 89 (2001) 2286.

[6] A. Azevedo, C. Chesman, S.M. Rezende, F.M. de Aguiar, X. Bian and S.S.P. Parkin, Phys. Rev. Lett. 76 (1996) 4837.

[7] N.S. Almeida and D.L. Mills, Phys. Rev. B 52 (1995) 13504. 


\section{Figure Captions}

(1) Fig. 1. Magnetization (in units of the saturation magnetization) versus applied field $H_{0}$ for (a) $N=3$, (b) $N=5$ and (c) $N=7$. The parameters are $H_{b l}=-1.0 \mathrm{kOe}, H_{u a}=0.5 \mathrm{kOe}, H_{b q}=0.1 \mathrm{kOe}$ and $\theta_{H}=\theta_{u a}=0^{\circ}$.

(2) Fig. 2. As in Fig. 1 but for $H_{b q}=0.7$ kOe.

(3) Fig. 3. Magnetization (normalized) versus applied field $H_{0}$ for three dif-

ferent values of angle $\theta_{H}$, taking $N=3, \theta_{u a}=0^{\circ}$ and other parameters as in Fig. 1. 


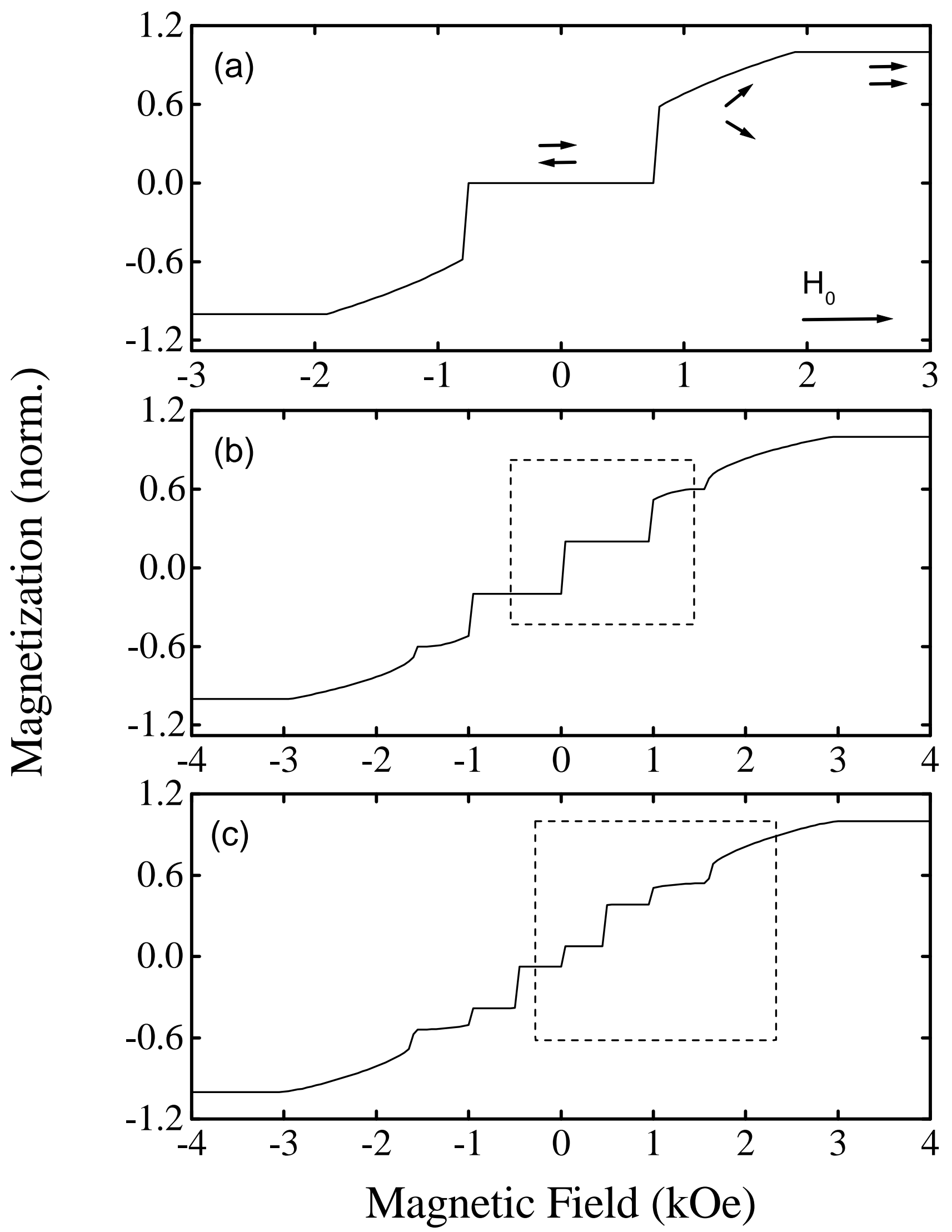



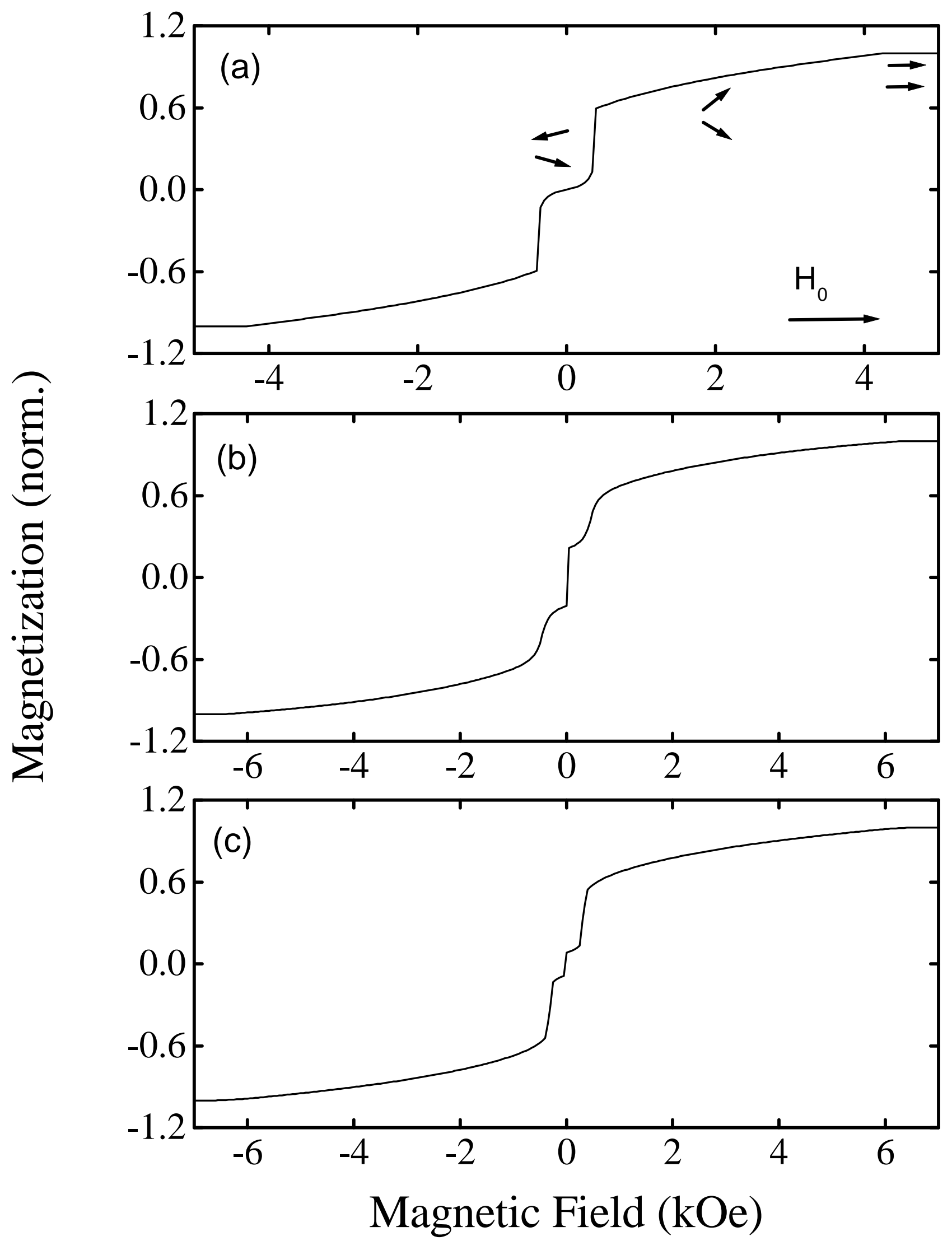


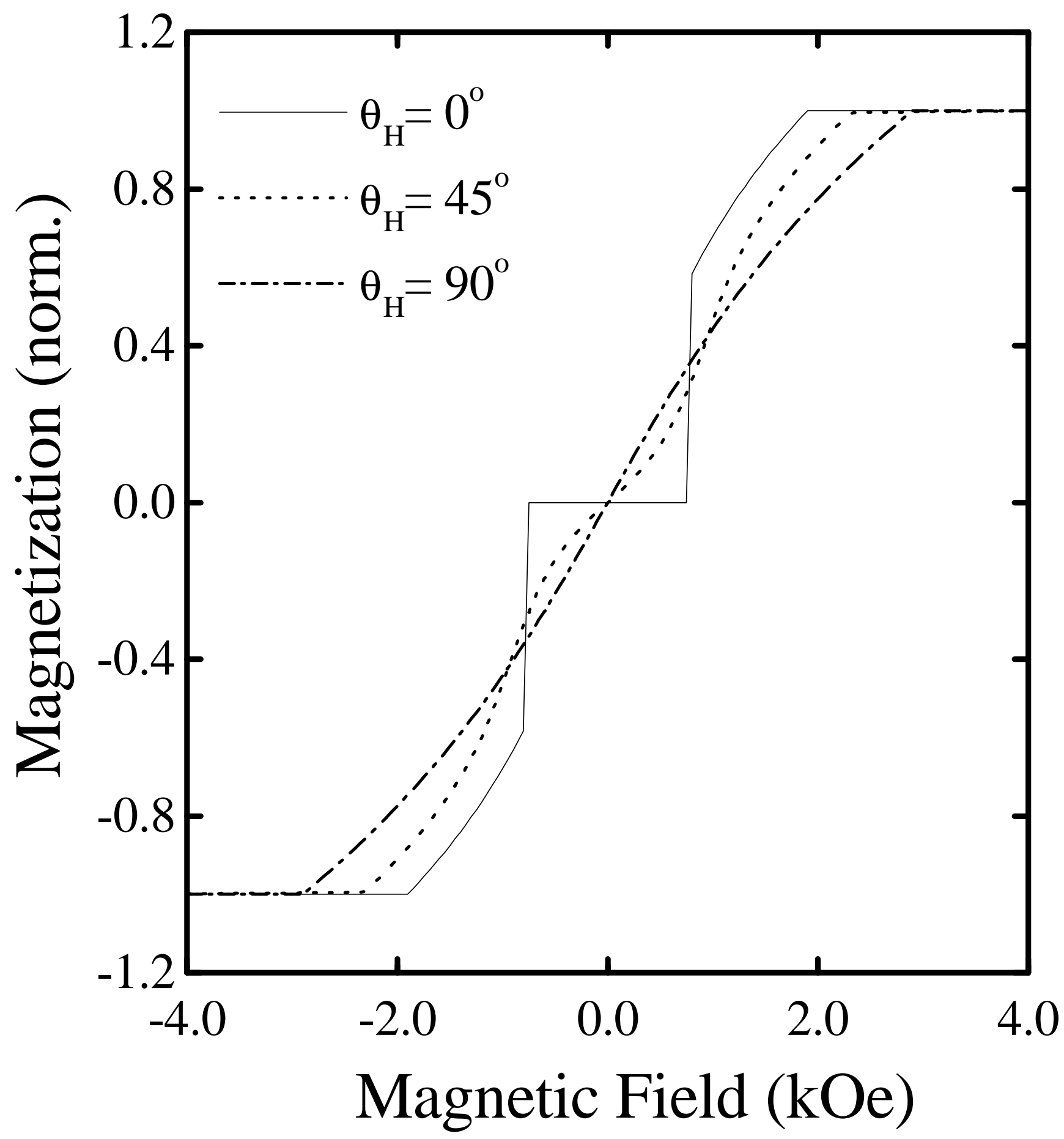

